Research Article

\title{
Optimization of Esophageal Ultrasound under Artificial Fish Swarm Algorithm and Its Adoption in Treatment of Ventricular Septal Defect
}

\author{
Zhi Li $\odot,{ }^{1}$ Guihe Chen $(1),{ }^{1}$ and Feng Wang $\oplus^{2}$ \\ ${ }^{1}$ Department of Cardiothoracic Surgery, The First People's Hospital of Huaihua, Huaihua 418000, Hunan, China \\ ${ }^{2}$ Department of Ultrasound, The First People's Hospital of Huaihua, Huaihua 418000, Hunan, China \\ Correspondence should be addressed to Zhi Li; 15110473063@stumail.sdut.edu.cn
}

Received 9 September 2021; Revised 31 October 2021; Accepted 5 November 2021; Published 24 November 2021

Academic Editor: M Pallikonda Rajasekaran

Copyright (C) 2021 Zhi Li et al. This is an open access article distributed under the Creative Commons Attribution License, which permits unrestricted use, distribution, and reproduction in any medium, provided the original work is properly cited.

\begin{abstract}
This work was aimed at exploring the adoption value of the optimized and upgraded esophageal ultrasound in the treatment of patients with ventricular septal defect (VSD) by artificial fish swarm algorithm. A model was built based on artificial fish swarm algorithm. A random ultrasonic optical signal in the database was decomposed several times and sparsity was optimized to complete partial optimization, which was then extended to global optimization. A total of 100 patients with ventricular septal defect were divided into control group who underwent cardiopulmonary bypass under the guidance of three-dimensional thoracic ultrasound and experimental group of ventricular septal defect occlusion under the guidance of esophageal ultrasound based on artificial fish swarm algorithm. The results showed that the number of successful cases in the experimental group was 12 cases of perimembranous type, 10 cases of septal type, 7 cases of simple membranous type, 13 cases of muscular type, 4 cases of subdry type, and 2 cases of ridge type. The average length of operation after surgery was 70.65 minutes, the average length of ventilator ventilation was 125.8 minutes, and the average length of intensive care unit was 377.9 minutes. The average length of hospital stay after surgery was 5.6 days, and the average total length of hospital stay was 8.2 days, which were better than the control group in many aspects, with statistical significance $(P<0.05)$. In short, the artificial fish swarm algorithm for esophageal ultrasound-guided ventricular septal defect closure had short operation time and good postoperative effect, which was of high application value in the clinical treatment of patients with ventricular septal defect.
\end{abstract}

\section{Introduction}

According to statistics, heart ventricular septal defect is second only to atrial septal defect in adult congenital heart disease. It refers to the abnormal development of the tissue separating the left and right ventricles during embryo, resulting in defects or holes $[1,2]$. Generally, patients with large defects will suffer from stunting, fatigue, shortness of breath, dyspnea, and other clinical symptoms. If it is not treated in time, subsequent serious diseases such as heart failure may result $[3,4]$. Clinically, three-dimensional transthoracic ultrasound is usually used for pathological diagnosis. In the diagnosis of obesity, emphysema, and other patients, however, this examination method has great limitations, which will affect the quality of ultrasonic image [5].
In the traditional ultrasonic detection, there are many defects in the optical signal, but there are also some redundant interference noises. Ordinary pulse interference noise can be removed by simple filtering algorithm. However, due to the noise emitted by some scattered grains or special materials, it is difficult to extract the defect information by using traditional algorithms, thus affecting the subsequent signal processing $[6,7]$. With the development of science and technology, transesophageal ultrasound examination gradually goes into the public vision. It involves the insertion of an esophageal probe into the patient's body from the esophagus, which is attached to the back wall of the heart [8-10]. In this way, it can avoid some interference tissue and intuitively detect the internal structure of the heart, which is helpful for the diagnosis of the disease. 
Artificial fish swarm algorithm is a new optimization algorithm based on simulated fish swarm behavior proposed by Li et al. in 2002. The basic idea is that the most fish in a body of water are at the most nutrient-rich parts of the body of water. Based on this characteristic, foraging behavior of fish shoal is imitated so as to realize global optimization [11-13]. The application of artificial fish swarm algorithm in esophageal ultrasound examination can find the optimal data in signal decomposition with high efficiency, optimize the operation framework, and improve the stability $[14,15]$. Moreover, it has good anti-interference ability, which plays an important role in improving the image quality of esophageal ultrasound $[16,17]$.

In this experiment, the artificial fish swarm algorithm was used to establish the esophageal model. By comparing a series of indexes of the two groups of patients, the application value of the esophageal ultrasound-guided blocking technique based on artificial fish swarm algorithm in the clinical treatment of VSD was comprehensively evaluated, expected to provide a more reliable basis for the clinical treatment of VSD.

\section{Materials and Methods}

2.1. Research Objects. A total of 100 patients who were admitted to hospital from May 2019 to December 2020 and needed to undergo ventricular septal defect surgery were randomly divided into control group and experimental group, with 50 patients in each group. The patients in control group ranged in age from 18 to 40 years, with an average age of 27.7 years. Three-dimensional transthoracic ultrasoundguided cardiopulmonary bypass was used to establish the operation. The patients in experimental group were with an average age of 28.2 years, who underwent VSD occlusion under the guidance of esophageal ultrasound based on artificial fish swarm algorithm. Inclusion criteria are as follows: (i) patients with cardiac function grades I-II; (ii) patients with no history of thoracic surgery; (iii) patients without other organic lesions; (iv) patients without esophageal and gastric ulcers one week before surgery; (v) patients with complete clinical and imaging data. Exclusion criteria are as follows: (i) patients with a history of thoracic surgery or with other organ diseases; (ii) patients who lacked compliance and did not cooperate with the examination; (iii) patients with incomplete clinical data; (iv) patients with pharyngeal or esophagus lesions. This experiment had been approved by the Ethics Committee of hospital. All relevant matters of the experiment had been informed to the patients themselves and their families, and informed consent had been signed.

\subsection{Three-Dimensional Transthoracic Ultrasound} Examination. The patient in the control group was placed in the left decubitus position, and the probe was placed beside the sternum. The probe was rotated every $1-3^{\circ}$ in the left ventricular long axis, atrial septum, ventricular septum, two groups of atrioventricular valves and pulmonary vein section, five apical chambers, and parasternal region. Multiple sections of the heart with different positions, angles, sizes, and shapes were obtained on the scanner. By continuous rotation in the plane of $0-180^{\circ}$, the long and short axial sections of the ventricular septal defect were obtained from multiple angles, so that the location of the ventricular septal defect was observed from multiple angles (Figure 1).

2.3. Surgery to Establish Cardiopulmonary Bypass. The patients in the control group received intramuscular injection of $0.01 \mathrm{mg} / \mathrm{kg}$ atropine and intravenous injection of $0.125 \mathrm{mg} / \mathrm{kg}$ midazolam 30 minutes before surgery, and anesthesia induction was performed with $3 \mu \mathrm{g} / \mathrm{kg}$ fentanyl, $0.1 \mathrm{mg} / \mathrm{kg}$ vecuronium bromide, and $1.25 \mathrm{mg} / \mathrm{kg}$ ketamine. After intubation, anesthesia machine was connected, and intermittent positive pressure ventilation was performed. The periosteum of the sternum was cut along the median line with an electric knife from the sternum notch, down to about $5 \mathrm{~cm}$ below the xiphoid process. Then, the pericardium was cut lengthwise midline between the reflexed part of the aorta and the diaphragm, and one incision was made on both sides of the lower incision. The pericardial margin was sutured together with the soft tissue outside the sternum on both sides, and the sternum was fixed separately to expose the heart. Inspections were made on each atrium, ventricle, and vein for size, tension, tremor, and any local aberrations. Patients were given a routine half-dose of heparin and heparinized to initiate cardiopulmonary bypass. First, a vena cava cuff was applied, and two concentric purse-bag sutures were made with No.7 silk thread at the end of the ascending aorta, with heparin injected. A small incision was made with a small round knife in the middle of the purse. At the same time when the round knife was put away, an artery intubation was inserted into the incision of the ascending aorta and the artificial heart-lung machine was connected. Then, a purse line was sewed into the right atrium and the right chamber. After incision, the catheter was inserted into the superior and inferior vena cava and then connected with the artificial heart-lung machine. A pad suture was made at the root of the ascending aorta. After draining of the gas from the needle with the cold cardioplegia, the catheter was inserted into the ascending aorta through the middle of the suture line, and the perfusion device was connected. A long suture line was made at the root of the right superior pulmonary vein where it connected to the left atrium. After small incision, the drainage tube was inserted into the left atrium and connected to the artificial heart-lung machine. After it was confirmed that all the tubes were correct, extracorporeal circulation was performed, and the superior and inferior vena cava and ascending aorta were blocked a few minutes later. $10 \mathrm{mg} / \mathrm{kg}$ of cold cardioplegia was infused into the aortic root perfusion tube. The surface of the heart was cooled with ice saline at $4^{\circ} \mathrm{C}$. At that point, the heart stopped rapidly and cardiopulmonary bypass was initiated. After the operation, the aorta was opened, and the upper and lower lumen occlusion belts were opened for parallel circulation until the heart automatically rebounded. Then, the machine was stopped, heparin was neutralized, potassium was supplemented to replenish blood volume, and extubation was done after the condition was stable. 


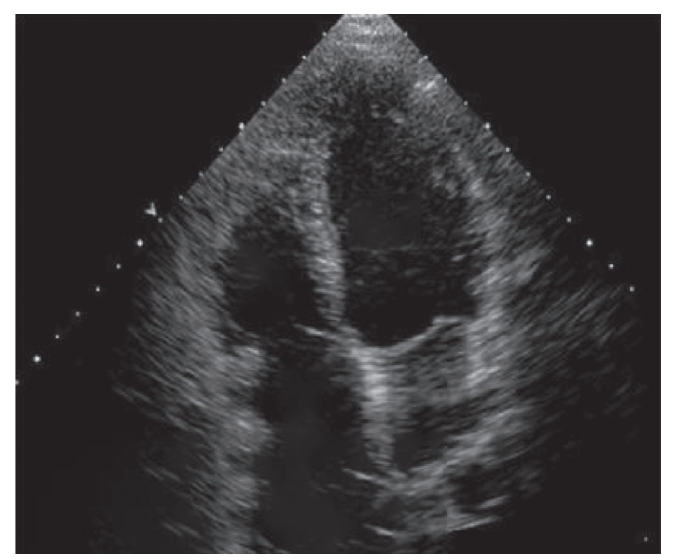

(a)

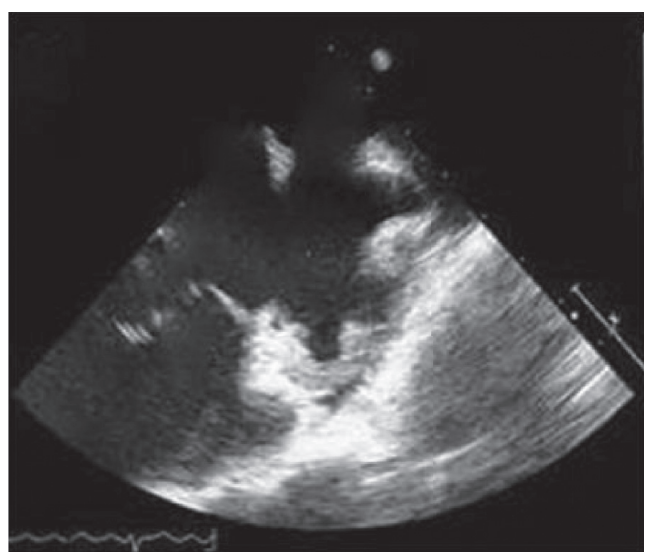

(b)

Figure 1: Image of apical four-chamber (a) and left ventricular short-axis view (b).

2.4. Ultrasonography of the Esophagus. Patients in the experimental group were supine and treated with general anesthesia through nasal endotracheal intubation, and the anesthesia drugs were the same as extracorporeal circulation. The esophagus ultrasonic probe was placed into the esophagus, and the left ventricular long axis, atrial septum, ventricular septum, two groups of atrioventricular valves, and the section of pulmonary vein, namely, the four chambers of the heart apex, were observed in the central part of the esophagus. In this way, the size and location of the ventricular septal defect, the length of connective tissue between the defect and the atrium and ventricle, and the regurgitation of the right valve were determined. Rotation of the probe to $30-45^{\circ}$ revealed a short axial section of the aortic root and the passage of the left ventricular pump to the artery, so that the length of the defect from the aortic valve was assessed, which was used to confirm its operational status and valve orifice regurgitation. Probe at $60-75^{\circ}$ can show the right ventricular pump flow to the artery and the junction between the right ventricle and the pulmonary artery, so as to determine the location and size of the defect, the availability of the right ventricular pump flow to the artery, and the presence of pulmonary valve stenosis. The probe at $120^{\circ}$ can show the passage of the left ventricular pump to the artery and the junction between the left ventricle and the pulmonary artery. Therefore, the length of the defect site from the aortic valve and the obstruction of the left ventricular pump blood flow channel to the artery were determined to assess the aortic valve regurgitate, as presented in Figure 2.

2.5. Ventricular Septal Defect Closure. In the experimental group, a small incision of about $3-4 \mathrm{~cm}$ was made at the lower sternum and xiphoid process in the supine position to separate the xiphoid process. The sternum was cut along the midline with an electric knife, and the heart sac was cut and suspended to expose the exterior of the right ventricle. The free wall of the right ventricle was suspended on both sides to make two purses, and the right ventricle purses were cut open and loaded into the guidance wire. Patients were given a routine half- amount of heparin heparinization. Under the guidance of esophageal ultrasound, the proximal end of the guide wire passed through the ventricular septal defect and entered the channel through which the right ventricular pump blood flew to the artery. Then, the puncture needle was removed and inserted into the dilating vessel sheath along the guide wire. The guide wire was withdrawn, and the conveying device and the heparinized occluder were placed into the dilated vessel sheath one by one. The blocker released the disk in turn in the left chamber of the defect and then retracted slightly, which then released the right ventricle disc in the right ventricle, repeated for several times. Esophageal ultrasound was used to examine whether there was residual shunt at the interventricular septum and whether there was valve regurgitation. The plugging device was adjusted to the appropriate position, and the conveying device was released after being fixed. The purse was tied in a knot, heparin was neutralized, and the chest was stopped layer by layer and closed.

\subsection{Artificial Fish Swarm Algorithm}

2.6.1. Construction of Artificial Fish Swarm Algorithm Model. In the decomposition process of optical signals, one of the most difficult steps is the calculation of the quantity product during each decomposition. It has large computation, high redundancy index, and low optimization efficiency. The content of artificial fish swarm algorithm is conducting single or partial optimization first, so as to realize overall optimization. It can optimize the maximum value and minimum value in a certain part of the operation efficiently, making the whole operation stable, so as to effectively overcome the problem of excessive calculation. The construction process of artificial fish swarm algorithm model is as follows.

First, it is supposed that $M=\left\{m_{1}, m_{2}, \ldots, m_{\lambda}\right\}$ is the atomic and molecular database for ultrasonic optical signal processing, where $m_{\lambda}$ is the atom with the property $\lambda$. After normalization of it, $\left\|m_{\lambda}\right\|=1$. A signal $t$ to be decomposed is selected from the database $M=\left\{m_{1}, m_{2}, \ldots, m_{\lambda}\right\}$, which is optimized as follows: 


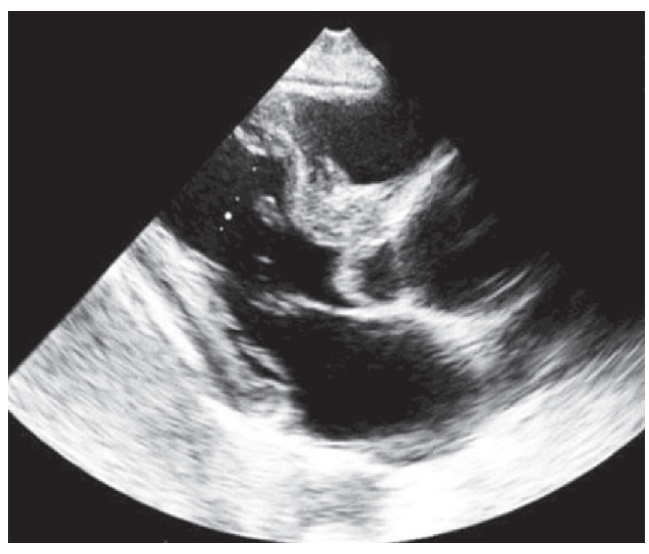

(a)

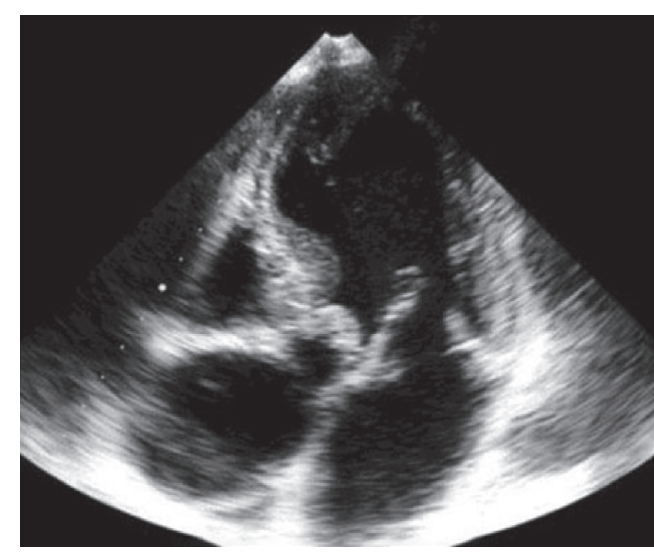

(b)

FIgURE 2: Apical four-chamber view (a) and short-axis view of aortic root (b).

$$
f(t)=\min \left\|m-\sum_{p=1}^{n} r_{i} m_{i}\right\| .
$$

In equation (1), $m_{i}$ is the $p$-th basic signal, and $r_{i}$ is its correlation decomposition coefficient. In the decomposition process, the biggest difficulty is the need to use several inner product operations, which requires a large amount of calculation and a high redundancy index. Therefore, the artificial fish swarm algorithm is selected to realize the construction of the model and the improvement of work efficiency.

It is assumed that the length of the signal $t$ to be decomposed is $A$; a data corresponding to $t$ is selected from the database to meet the following requirements:

$$
\left|\left[t, m_{\lambda p}\right]\right|=\sup \left|\left[t, m_{\lambda}\right]\right| .
$$

$\mathrm{O}^{1} t$ is the difference between the actual observation value and the fitted value after the original optical signal and the optimized data are paired. To implement signal decomposition, the equation is as follows:

$$
f^{\prime}(t)=\left[t, m_{\lambda}\right] m_{\lambda}+O^{\prime} t
$$

$s$ decompositions are performed on the difference between the actual observation value and the fitted value. When the requirements of equation (4) are met, equation (5) is meaningful.

$$
\begin{aligned}
\left|\left[O^{s} t, m_{\lambda}\right]\right| & =\sup \left|\left[O^{s} t, m_{\lambda p}\right]\right|, \\
O^{s} t & =\left[O^{s} t, m_{\lambda p}\right] m_{\lambda}+O^{s+1} t .
\end{aligned}
$$

In equation (5), the difference $O^{1} t$ between the actual observation value and the fitted value after $c$-th decompositions is expressed as follows:

$$
f^{\prime \prime}(t)=\sum_{s=1}^{c}\left[O^{s} t, m_{\lambda p}\right] m_{\lambda}+O^{c} t .
$$

When $c$ increases, $\left\|O^{c} t\right\|$ will be in a decreasing state until it decreases to 0 . Then, the improvement of the existing structure of the ultrasonic optical signal is completed, which is expressed as follows:

$$
F(t)=\sum_{s=1}^{c}\left[O^{s} t, m_{\lambda p}\right] m_{\lambda} .
$$

The properties of ultrasonic optical signals are similar to Gaussian functions. When the signals are decomposed, $m_{\lambda}$ needs to meet the requirements of normal distribution, which is expressed as follows:

$$
m_{\lambda}=\frac{1}{\sqrt{\theta}} e^{-\pi(\vartheta-\phi / \theta)^{2}} \cos (\phi x+\eta) .
$$

Based on the theoretical concept of normal distribution, an artificial fish swarm algorithm model is built to find the optimal data. When $\lambda=\{\theta, \vartheta, \phi, \eta\}$ is taken as a time-frequency distribution parameter, the set range of all values contained in a specific requirement is expressed as follows:

$$
\left\{\begin{array}{l}
\theta \in(1, A], \\
\vartheta \in[0, A], \\
\phi \in[0,2 \pi), \\
\eta \in[0.2 \pi] .
\end{array}\right.
$$

Based on the abovementioned time-frequency distribution parameters, an artificial fish swarm algorithm model is built, which is expressed as follows:

$$
\begin{aligned}
& \left|\left[O^{c} t, m_{\lambda}\right]\right|, \\
& m_{\lambda}=\frac{1}{\sqrt{\theta}} e^{-\pi(\vartheta-\phi / \theta)^{2}} \cos (\phi x+\eta) .
\end{aligned}
$$

The application of artificial fish swarm algorithm model can solve the disadvantages of large amount of calculation and high redundancy index caused by inner product operation and effectively improve the efficiency of ultrasonic optical signal processing. 
2.6.2. Decomposition of Ultrasonic Optical Signal. After the artificial fish swarm algorithm model is constructed, it is used to realize overall optimization and effectively complete the decomposition and processing of ultrasonic optical signals. A single factor in an artificial fish swarm is described by a set of vectors $E=\left\{e_{1}, e_{2}, \ldots, e_{n}\right\}$, where $e_{i}$ is set as the vector to be optimized, and the food content of the fish swarm currently prescribed is expressed as follows:

$$
G=f\left(e_{i}\right) .
$$

$G$ is the ideal function to be achieved by the fish swarm, and the distance $h_{i}$ between a single factor of the fish swarm and a single factor is expressed as follows:

$$
h\left(e_{i}, e_{k}\right)=\sqrt{\sum_{i=1}^{n}\left(e_{i}-e_{k}\right)^{2}} .
$$

The sight range of a fish swarm's single factor and adjacent factors is expressed as follows:

$$
\text { Adjoin }=\left\{e_{k} \mid 0<h_{i}, k \leq \text { view }\right\}, \quad l=1,2, \ldots, n .
$$

In equation (13), $l$ is the total amount of individual factors in the fish swarm, and the individual factors in the fish swarm in a transition state are expressed as follows:

$$
e_{q}=\frac{\sum_{i=1}^{n} e_{i}(l)}{l} ; \quad l=1,2, \ldots, n .
$$

The position and length of the transfer of a single factor in the fish swarm are expressed as follows:

$$
e_{i+1}=e_{i}+\text { step } \cdot \frac{e_{k}(l)-e_{i}(l)}{\left\|e_{k}(l)-e_{i}(l)\right\|}, \quad l=1,2, \ldots, n .
$$

To ensure the stability of the calculation process, a single factor must be handled within a transferable range and must not exceed the corresponding range. If there is a mistake in one operation, it is necessary to perform the overall operation again until the optimal solution is searched.

2.7. Statistical Analysis. SPSS 24.0 was employed for data statistics and analysis. Mean \pm standard deviation $(x \pm s)$ was how measurement data were expressed, and percentage (\%) was how count data were expressed. The $t$-test was used when the experimental data conformed to normal distribution. $\chi^{2}$ test was used for univariate analysis of related factors, and logistic regression analysis was used for multivariate analysis. $P<0.05$ was considered statistically significant.

\section{Results}

3.1. Comparison of General Clinical Data of the Two Groups of Patients. Figure 3 below shows the comparison of general clinical data between two groups of patients. There were no substantial differences in age, sex ratio, height, and weight between the control group and the experimental group $(P>0.05)$.

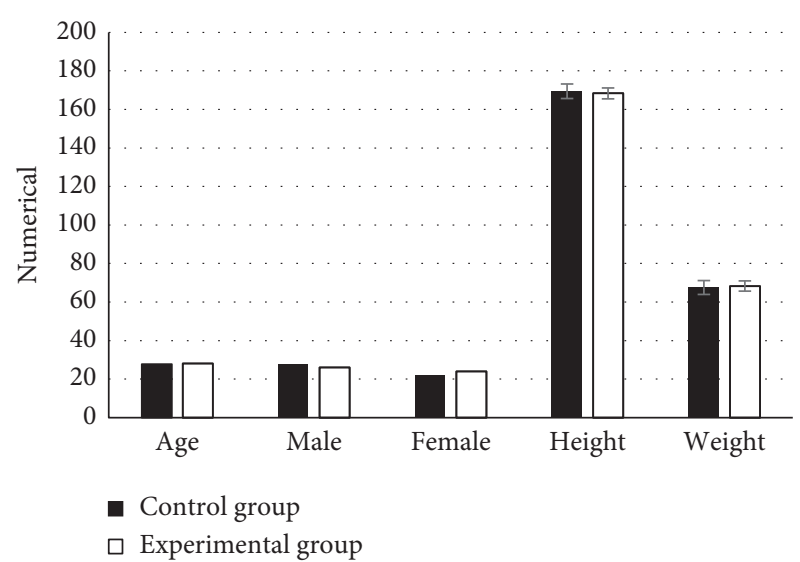

Figure 3: Comparison of general clinical data of the two groups of patients.

3.2. Comparison of the Number of Successful Operations between the Two Groups. Figure 4 shows that after three-dimensional transthoracic ultrasound-guided cardiopulmonary bypass was established in control group, there were 12 cases of perimembrane type of ventricular septal defect, of which 10 cases were successful; 10 cases of septal valve type, of which 8 cases were successful; 8 cases of simple membranous type, of which 6 cases were successful; 15 cases of muscular type, of which 12 cases were successful; 2 cases of substem type, of which 1 case was successful; 3 cases of crest type, of which 3 cases were successful. In the experimental group, there were 13 cases, 11 cases, 7 cases, 13 cases, 4 cases, and 2 cases of ventricular septal defect occlusion under the guidance of esophageal ultrasound based on artificial fish swarm algorithm. The number of successful cases were $12,10,7,13,4$, and 2 , respectively. The number of successful cases of ventricular septal defect closure guided by esophageal ultrasound based on artificial fish swarm algorithm was greatly higher than that in the control group, and the difference was considerable $(P<0.05)$.

3.3. Comparison of Preoperative and Postoperative Left Ventricular Status between the Two Groups. Figure 5 shows that the left ventricular size and the left ventricular end-diastolic volume were reduced to some extent between the control group and the experimental group at three days, one month, and three months after surgery, but the differences were not considerable compared with those before surgery $(P>0.05)$.

3.4. Comparison of Cardiac Function between the Two Groups of Patients before and after Surgery. In Figure 6, there was no considerable difference in left ventricular ejection fraction and left ventricular short-axis shorting rate in the four postoperative reexaminations between the control group and the experimental group $(P>0.05)$.

3.5. Comparison of the Number of Cases of Postoperative Pericardial Effusion and Arrhythmia between the Two Groups. Figure 7 shows that, in the control group, postoperative pericardial effusion occurred in 15 cases, incomplete right 


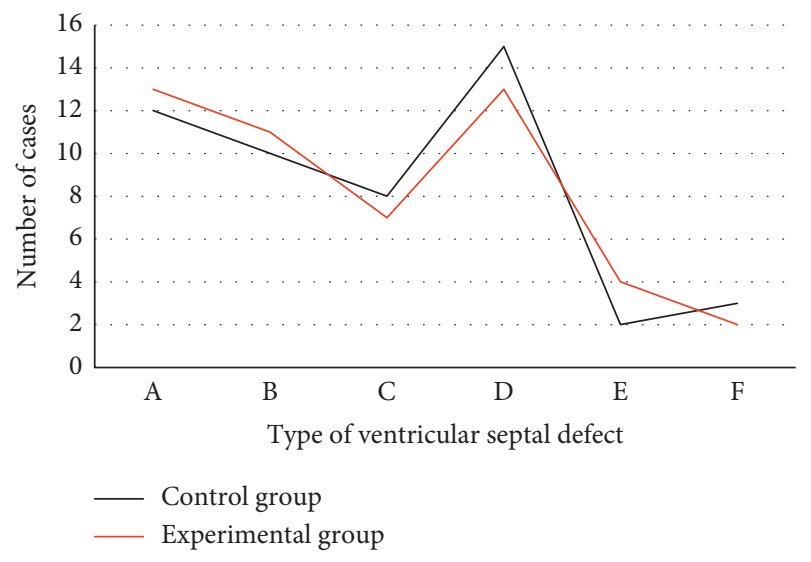

(a)

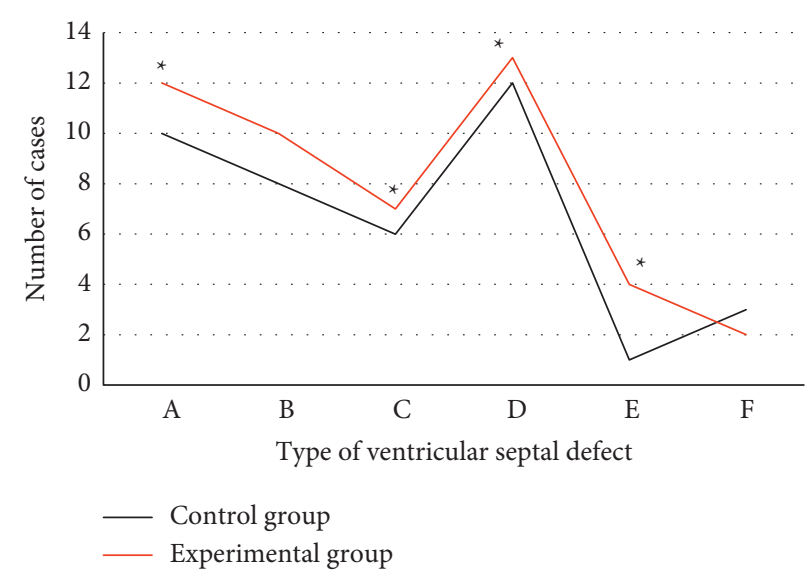

(b)

FiguRE 4: Comparison of the number of successful operations between the two groups: (a) the number of cases of ventricular septal defect types in the two groups; (b) the number of successful operations: (A) perimembrane type, (B) septal type, (C) simple membrane type, (D) muscle type, (E) subdry type, (F) ridge type. ${ }^{*}$ indicates that the number of successful operations in the experimental group was greatly different from the control group $(P<0.05)$.

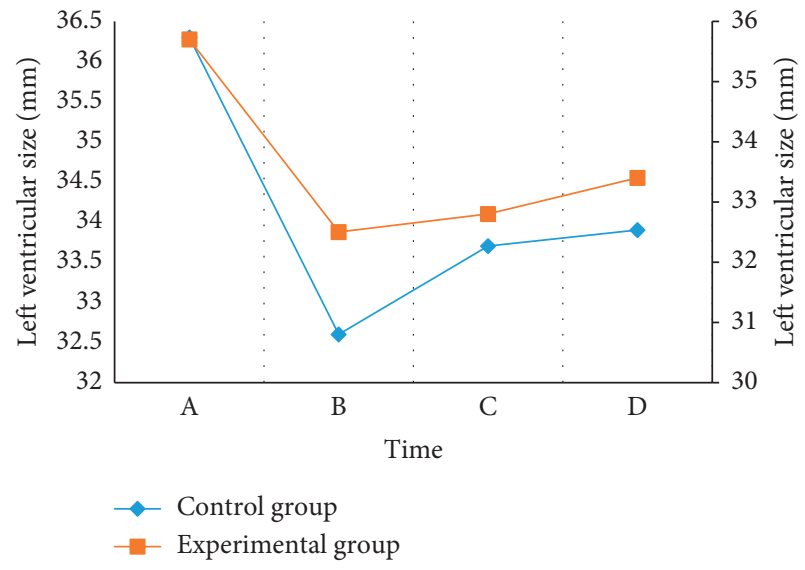

(a)

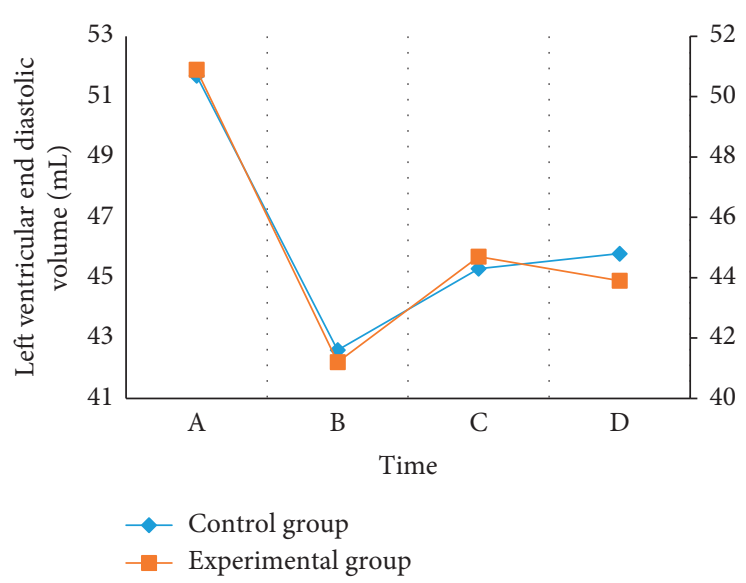

(b)

Figure 5: Comparison of left ventricular state before and after surgery between the two groups. (a) The comparison of the left ventricular size before and after surgery; (b) the comparison of the left ventricular end-diastolic volume before and after surgery: (A) before surgery, (B) three days after surgery, (C) one month after surgery, and (D) three months after surgery.

bundle branch block in 31 cases, complete right bundle branch block in 4 cases, left anterior branch block in 1 case, and III $^{\circ}$ atrioventricular block in 1 case. The number of these complications in the test group was $6,20,1,0$, and 0 , respectively. The number of cases of pericardial effusion and arrhythmia in experimental group was greatly inferior to that in control group, with statistical significance $(P<0.05)$.

3.6. Comparison of Duration of Operation, Duration of Ventilator Ventilation, and Duration of Intensive Care Unit between the Two Groups. Figure 8 shows that, in the control group, the average duration of operation was 157.88 minutes, the average duration of ventilator ventilation was 460.7 minutes, and the average duration of intensive care unit was 1187.6 minutes. In the experimental group, the mean operative duration was 70.65 minutes, the mean ventilator duration was 125.8 minutes, and the mean ICU duration was 377.9 minutes. The durations of operation, ventilator ventilation, and intensive care unit in experimental group were greatly shorter than those in control group, with statistical significance $(P<0.05)$.

3.7. Comparison of Hospitalization Days between the Two Groups. Figure 9 shows that the average length of hospital stay in the control group was 7.5 days, and the average total length of hospital stay was 13.8 days. The mean length of hospital stay in the experimental group was 5.6 days, and the mean total length of hospital stay was 8.2 days. The length of hospital stay in experimental group was greatly inferior to that in control group $(P<0.05)$. 


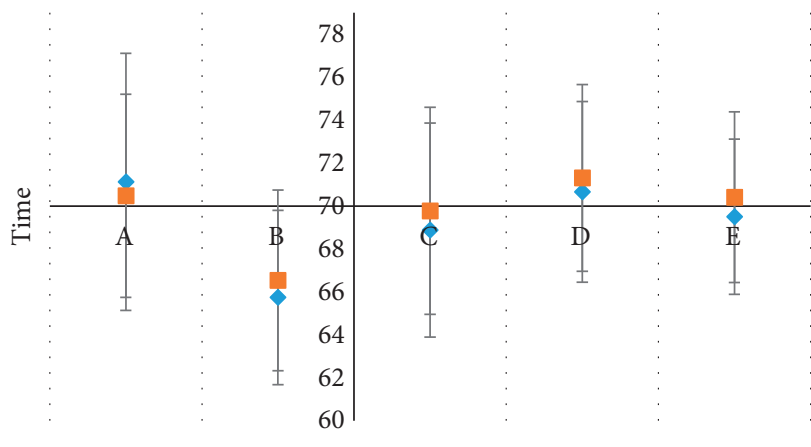

Left ventricular ejection fraction (\%)

- Control group

- Experimental group

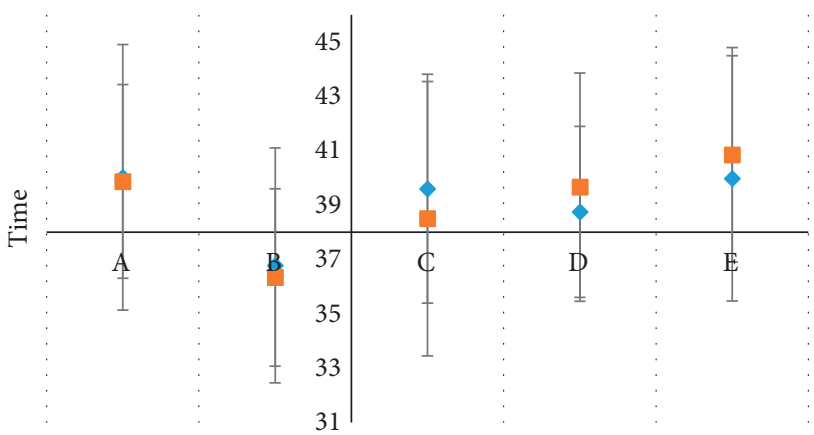

Left ventricular minor axis decurtation (\%)

$$
\begin{aligned}
& \text { Control group } \\
& \text { Experimental group }
\end{aligned}
$$

(a)

(b)

Figure 6: Comparison of cardiac function between the two groups of patients before and after surgery. (a) Comparison of the left ventricular ejection fraction before and after surgery, (b) comparison of the left ventricular short-axis shortening rate before and after surgery: (A) before surgery, (B) first review after surgery, (C) second review after surgery, (D) third review after surgery, and (E) fourth review after surgery.

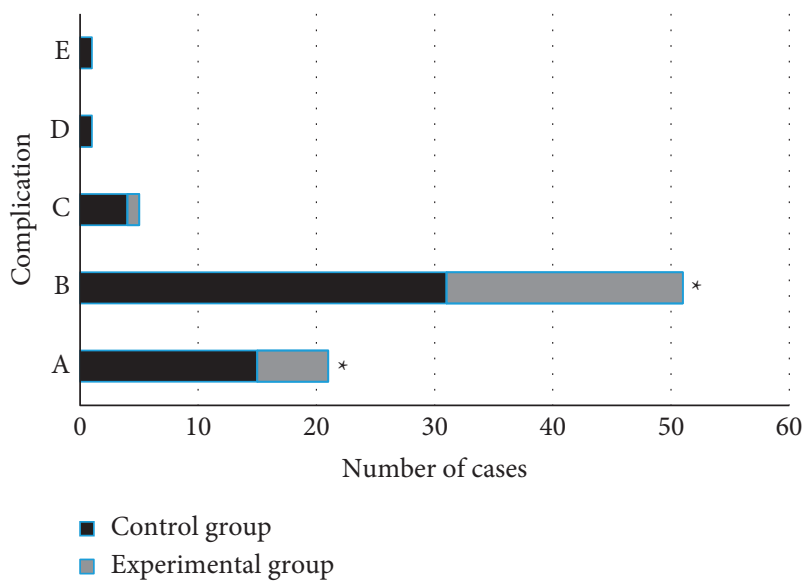

Figure 7: Comparison of the number of cases of postoperative pericardial effusion and arrhythmia between the two groups. (A) pericardial effusion, (B) incomplete right bundle branch block, (C) complete right bundle branch block, (D) left anterior branch block, (E) III $^{\circ}$ atrioventricular block. ${ }^{*}$ indicated that the number of patients with pericardial effusion and incomplete right bundle branch block in the experimental group was greatly different from that in the control group $(P<0.05)$.

3.8. Ultrasonic Images of Esophagus before and after the Application of Artificial Fish Swarm Algorithm. A patient, male, 22 years old, had shortness of breath and dyspnea for more than half a year. During routine esophageal ultrasound examination, due to the equipment level and other reasons, ultrasound image contrast was insufficient. There were redundant interference noise and poor spatial resolution, which brought some difficulties for the observation of the heart environment. The artificial fish swarm algorithm was applied in esophageal ultrasound to improve the decomposition speed of optical signals, and the searching signal results were more accurate and the imaging was clearer, as illustrated in Figure 10.

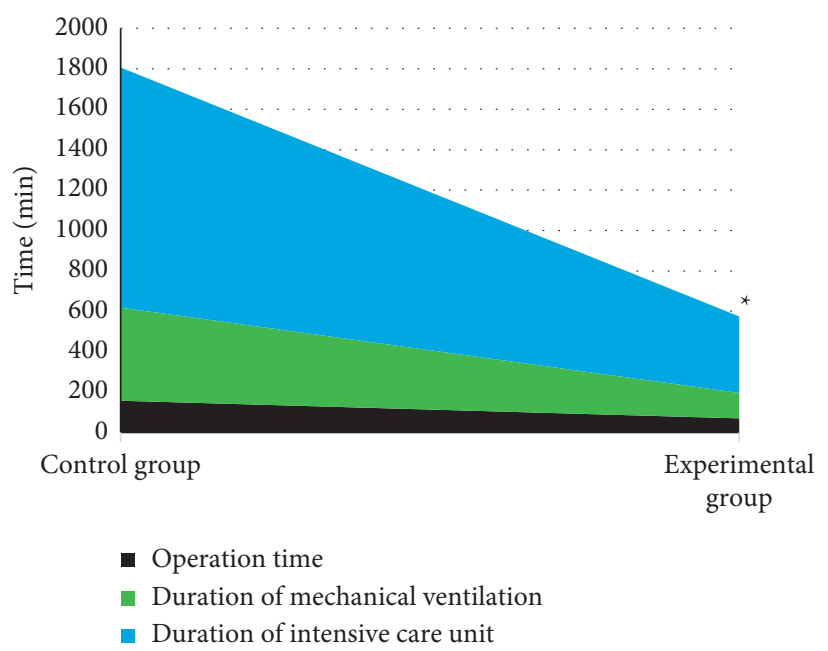

Figure 8: Comparison of the durations of operation, ventilator ventilation, and intensive care unit between the two groups. ${ }^{*}$ indicated that there were substantial differences in the durations of operation, ventilator ventilation, and intensive care unit in the experimental group compared with the control group $(P<0.05)$.

\section{Discussion}

An artificial fish swarm algorithm was selected in this experiment. Its essence was simulating the foraging, clustering, and tail-chasing behaviors of fish swarm by constructing an artificial fish model, so as to achieve overall optimization [18]. In this experiment, an atom was randomly selected from the database and optimized by maxima and minima, normalization, multiple decomposition, etc., to complete the optimization of single factor. Then, the artificial fish swarm algorithm model was built, and then the objective of global optimization was approached gradually through optical signal decomposition and processing in the designated range. The artificial fish swarm algorithm was applied to a 


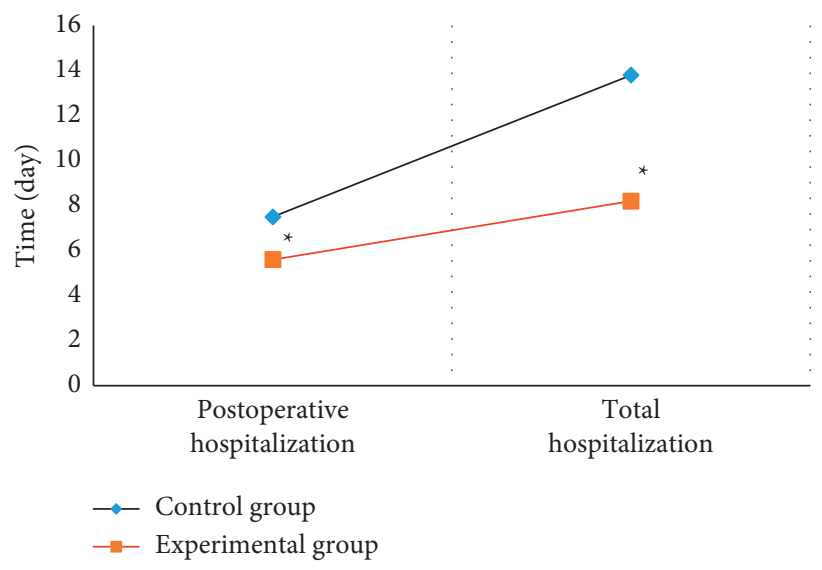

Figure 9: Comparison of hospitalization days between the two groups. ${ }^{*}$ indicated that the average length of postoperative hospital stay and the average total length of postoperative hospital stay in the experimental group were greatly different from those of control group $(P<0.05)$.

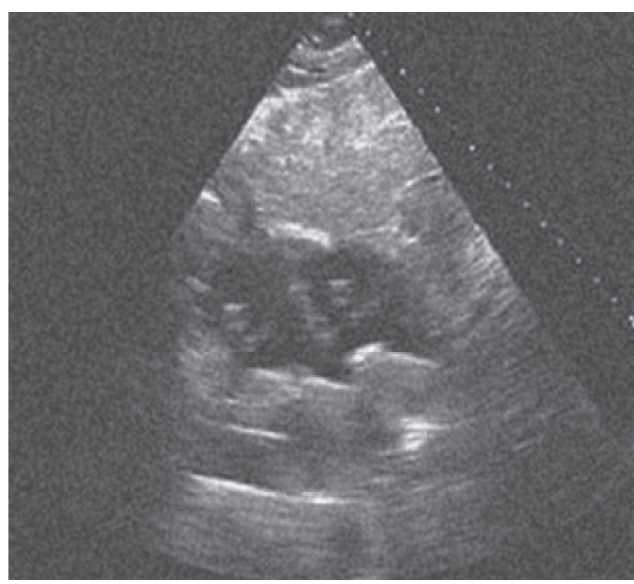

(a)

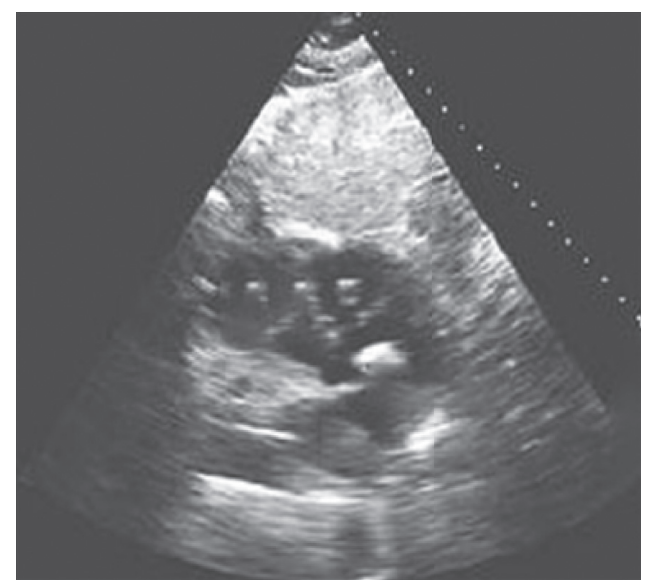

(b)

Figure 10: Original ultrasonic image of esophagus (a) and ultrasonic image of esophagus after the application of artificial fish swarm algorithm (b).

collection of 100 patients with ventricular septal defects requiring surgical treatment. The patients in control group were treated with three-dimensional transthoracic ultrasound-guided cardiopulmonary bypass, and those in experimental group were treated with artificial fish-based ultrasound-guided ventricular septal defect closure. The results showed that the number of successful cases in the experimental group was greatly higher than that in the control group $(P<0.05)$. The number of cases with pericardial effusion and arrhythmia after operation was inferior to that of control group, and the difference was notable $(P<0.05)$. Moreover, the durations of operation, ventilator ventilation, and intensive care unit were greatly shorter than those of the control group $(P<0.05)$. The length of hospitalization was greatly inferior to that of the control group $(P<0.05)$. In 2021, Yang et al. [19] pointed out that the occlusion of ventricular septal defect under the guidance of esophageal ultrasound had a high safety and feasibility because of shorter operation time and less intraoperative bleeding compared with traditional repair. In this study, the reliability of this statement was verified. The ventricular septal defect occlusion under the guidance of esophageal ultrasound based on artificial fish swarm was performed. It had short operation time, short hospitalization days, and good prognosis and was acceptable to patients' family members, which was of great significance in the treatment of ventricular septal defect.

\section{Conclusion}

In this study, the artificial fish swarm algorithm was used to optimize the traditional esophageal ultrasound, and 100 patients requiring surgical treatment of ventricular septal defects were collected as the research subjects. The results showed that the experimental group was better than the control group in terms of the number of successful operations, the number of cases with pericardial effusion and arrhythmia after operation, the duration of operation, the duration of ventilator ventilation, the duration of intensive care unit, and the length of hospital stay $(P<0.05)$. However, 
the sample size collected in this experiment was small, the length of hospitalization of patients was short, and the observation had certain limitations and one-sidedness. It is expected that further research in this direction will provide a more reliable basis for the clinical treatment of ventricular septal defect.

\section{Data Availability}

The data used to support the findings of this study are available from the corresponding author upon request.

\section{Conflicts of Interest}

The authors declare no conflicts of interest.

\section{References}

[1] B. H. Morray, "Ventricular septal defect closure devices, techniques, and outcomes," Interventional Cardiology Clinics, vol. 8, no. 1, pp. 1-10, 2019.

[2] L. Lopez, L. Houyel, S. D. Colan et al., "Classification of ventricular septal defects for the eleventh iteration of the international classification of diseases-striving for consensus: a report from the international society for nomenclature of paediatric and congenital heart disease," The Annals of Thoracic Surgery, vol. 106, no. 5, pp. 1578-1589, 2018.

[3] S. Omar, G. L. Morgan, H. B. Panchal et al., "Management of post-myocardial infarction ventricular septal defects: a critical assessment," Journal of Interventional Cardiology, vol. 31, no. 6, pp. 939-948, 2018.

[4] Y.-S. Cho, S. E. Park, S.-K. Hong, N.-Y. Jeong, and E.-Y. Choi, "The natural history of fetal diagnosed isolated ventricular septal defect," Prenatal Diagnosis, vol. 37, no. 9, pp. 889-893, 2017.

[5] L. P. Badano, P. Aruta, K. Nguyen et al., "Principali applicazioni dell'ecocardiografia tridimensionale nell'attuale pratica clinica [Current clinical applications of three-dimensional echocardiography]," Glomale Italiano di Caradiologia, vol. 20, no. 12, pp. 722-735, 2019.

[6] X. Bai, M. Hu, T. Gang, and Q. Rong, "A submerged optical fiber ultrasonic sensor using matched fiber bragg gratings," Sensors, vol. 18, no. 6, Article ID 1942, 2018.

[7] W. Y. Choi, S. W. Kwon, Y. H. Kim, K. C. Kang, and K. K. Park, "Single-shot near-field volumetric imaging system for optical ultrasound and photoacoustics using capacitive micromachined ultrasonic transducer without transmission mode," IEEE Transactions on Ultrasonics, Ferroelectrics, and Frequency Control, vol. 67, no. 6, pp. 1151-1158, 2020.

[8] A. B. Freitas-Ferraz, M. Bernier, R. Vaillancourt et al., "Safety of transesophageal echocardiography to guide structural cardiac interventions," Journal of the American College of Cardiology, vol. 75, no. 25, pp. 3164-3173, 2020.

[9] T. C. Wray, K. Schmid, D. Braude et al., "Safety of transesophageal echocardiography performed by intensivists and emergency physicians in critically ill patients with coagulopathy and thrombocytopenia: a single-center experience," Journal of Intensive Care Medicine, vol. 36, no. 1, pp. 123-130, 2021.

[10] X. Lei, X. Yang, and F. Wu, "Artificial fish swarm optimization based method to identify essential proteins," IEEE/ACM Transactions on Computational Biology and Bioinformatics, vol. 17, no. 2, p. 1, 2019.
[11] S. Xie, Z. Yu, and Z. Lv, "Multi-disease prediction based on deep learning: a survey," Computer Modeling in Engineering and Sciences, vol. 128, no. 2, pp. 489-522, 2021.

[12] M. Mostefa-Kara, L. Houyel, and D. Bonnet, "Anatomy of the ventricular septal defect in congenital heart defects: a random association?" Orphanet Journal of Rare Diseases, vol. 13, no. 1, p. 118, 2018.

[13] U. Radhakrishna, S. Albayrak, R. Zafra et al., "Placental epigenetics for evaluation of fetal congenital heart defects: ventricular Septal Defect (VSD)," PLoS One, vol. 14, no. 3, Article ID e0200229, 2019.

[14] T. Buck, L. Bösche, and B. Plicht, "Echtzeit-3-D-Echokardiographie zur Schweregradbeurteilung von Herzklappenvitien," Herz, vol. 42, no. 3, pp. 241-254, 2017.

[15] S. Montis, D. Sirigu, A. Marini et al., "L'ipnosi nell'ecocardiografia transesofagea. L'esperienza di un Centro di Cardiologia Pediatrica e delle Cardiopatie Congenite [Hypnosis in transesophageal echocardiography. The experience in a Pediatric Cardiology and Congenital Heart Disease Unit]," Glomale Italiano di Caradiologia, vol. 20, no. 11, pp. 651-657, 2019.

[16] T. Gluck, M. Kravchik, S. Chocron, Y. Elovici, and A. Shabtai, "Spoofing attack on ultrasonic distance sensors using a continuous signal," Sensors, vol. 20, no. 21, Article ID 6157, 2020.

[17] Z. Kou, F. Yang, J. Wu, and T. Li, “Application of ICEEMDAN energy entropy and AFSA-SVM for fault diagnosis of hoist sheave bearing," Entropy, vol. 22, no. 12, Article ID 1347, 2020.

[18] S. Schubert, M. Kelm, N. R. Koneti, and F. Berger, "First European experience of percutaneous closure of ventricular septal defects using a new CE-marked VSD occluder," EuroIntervention, vol. 15, no. 3, pp. e242-e243, 2019.

[19] H. Yang, J. Mu, Y. Zhao, Z. Chen, H. Song, and J. Liu, “Transesophageal echocardiography guided closure of ventricular septal defect with 2 occluders from different incisions simultaneously," Medicine (Baltimore), vol. 100, no. 19, Article ID e23854, 2021. 